\title{
A chemical ecogenomics approach to understand the roles of secondary metabolites in fungal cereal pathogens
}

\author{
Yit-Heng Chooi* and Peter S. Solomon \\ Plant Sciences Division, Research School of Biology, The Australian National University, Canberra, ACT, Australia
}

\author{
Edited by: \\ Jonathan Mark Palmer, USDA Forest \\ Service, USA \\ Reviewed by: \\ Armen Trchounian, Yerevan State \\ University, Armenia \\ Gelhaye Eric, Université de Lorraine, \\ France \\ *Correspondence: \\ Yit-Heng Chooi, Plant Sciences \\ Division, Research School of Biology, \\ The Australian National University, \\ Building 134, Linnaeus Way, Canberra, \\ Acton, ACT 2601, Australia \\ e-mail:yh.chooi@anu.edu.au
}

Secondary metabolites (SMs) are known to play important roles in the virulence and lifestyle of fungal plant pathogens. The increasing availability of fungal pathogen genome sequences and next-generation genomic tools have allowed us to survey the SM gene cluster inventory in individual fungi. Thus, there is immense opportunity for SM discovery in these plant pathogens. Comparative genomics and transcriptomics have been employed to obtain insights on the genetic features that enable fungal pathogens to adapt in individual ecological niches and to adopt the different pathogenic lifestyles. Here, we will discuss how we can use these tools to search for ecologically important SM gene clusters in fungi, using cereal pathogens as models. This ecological genomics approach, combined with genome mining and chemical ecology tools, is likely to advance our understanding of the natural functions of SMs and accelerate bioactive molecule discovery.

Keywords: fungal secondary metabolites, ecological genomics, chemical ecology, genome mining, plant pathogen

\section{INTRODUCTION}

The interactions of fungal plant pathogens with their hosts are highly complex and involve both secondary metabolites (SMs) and small secreted proteins as pathogenicity factors (often defined as effectors). The role of SMs in mediating the virulence of fungal plant pathogens is increasingly being recognized (Mobius and Hertweck, 2009; Collemare and Lebrun, 2011). These fungal SMs facilitate infection by altering host cell structure or function via diverse mode of actions. Some of these SMs are host-selective toxins (HSTs) while others are nonhost-selective general phytotoxins. Some classic examples of small molecule HSTs are found amongst the phytopathogens in the Dothideomycete class (Stergiopoulos et al., 2013; MuriaGonzalez et al., 2014), including victorin, T-toxin, and HC-toxins. Other well-known examples of non-host selective phytotoxins include cercosporin, tentoxin, beticolin, depudecin, AALtoxin, deoxynivalenol (DON) etc., each with different mode of actions.

The increasing number of phytopathogen genome sequences has revealed a large number of uncharacterized SM gene clusters in these fungi, particularly within the Dothideomycete but also other ascomycete pathogens such as Magnaporthe and Fusarium species. This indicates that we have merely scratched the surface of the SM repertoire in phytopathogens. Many SM gene clusters that encode the production of small molecules that have a role in host interactions are likely waiting to be discovered from these genomes. These phytopathogens SMs are equally likely to act as suppressors of other competing microbes that shared the ecological niches as well as deterrents of herbivores and fungivores (insects and other animals).

From a natural product discovery perspective, the flood of phytopathogen genome sequences presents an exciting opportunity for genome mining of bioactive molecules. Since eukaryotes, from fungi, plants to human beings, share many core biochemical pathways, many SMs that are targeted at plants and other eukaryotic microorganisms are likely to interact with macromolecules in human as well. Indeed, many molecules with human biological targets have been discovered from plantassociated fungi. Notable examples include, squalene synthase inhibitors zaragozic acids (Bergstrom et al., 1995), actin polymerization inhibitors cytochalasans (Scherlach et al., 2010), calmodulin inhibitors ophiobolins (Au et al., 2000), and various histone deacetylase inhibitors including depudecin, apicidin, and HCtoxin (Walton, 2006). These molecules have high clinical relevance and often serve as drug leads in drug discovery programs. Phytopathogens, which have to compete with other microbes in the environment, are also potential source of antimicrobial compounds. For example, aspergillomarasmine A, which was recently shown to be a promising metallo- $\beta$-lactamase inhibitor to combat antibiotic resistant bacteria (King et al., 2014), was originally isolated from the cereal pathogen Pyrenophora teres (Haenni et al., 1965). Despite that the biological targets of some of these bioactive SMs are known, the biological roles and ecological functions of these SMs often remain enigmatic.

It has been recognized that the chemical ecology research can advance the discovery of bioactive molecules (Caporale, 1995). With the new next-generation genomic tools now at our disposal, we believe it is time to explore the synergy of ecological genomics and chemical ecology for advancing the understanding of the SM functions in phytopathogens. We termed this emerging integrated approach chemical ecogenomics. Combined with the increasingly mature genome mining tools and heterologous systems for expression of fungal SMs, this approach is likely to greatly accelerate the discovery of SM virulence factors and bioactive molecules. Similar strategies have been proposed for the study of insect pheromones (Tittiger, 2004), the role of fungal SMs in interactions with animals (Kempken and Rohlfs, 2010; Rohlfs and Churchill, 2011), and for natural product discovery in 
endophytic fungi (Kusari et al., 2012), coprophilous fungi (Bills et al., 2013) and Gammaproteobacteria (Vizcaino et al., 2014). Here, we would like to take some of these ideas one step further and to propose a basic chemical ecological framework for the study of SMs in phytopathogens, in particular, in cereal pathogens (Figure 1).

\section{CHEMICAL ECOGENOMICS - THE NEXUS OF CHEMICAL ECOLOGY AND ECOLOGICAL GENOMICS}

Ecological genomics seeks to understand the function of genes and genome in biotic and abiotic interactions among organisms and their natural environments (Ungerer et al., 2008). Functional genomics tools, including comparative genomics, transcriptomics, and proteomics, are used to study the genome, transcriptome, and proteome dynamics in an ecological context. These studies often identify candidate genes that are important to a given ecological interactions or environmental niche. The identified genes are subjected to further functional verification. Similar studies focusing specifically on host-pathogen interactions in both human and plant pathogenic fungi are also often referred as pathogenomics (Pompe etal., 2005; Schmidt and Panstruga, 2011).

On the other hand, chemical ecology is the study of small molecules that mediate the biotic and abiotic interactions of organisms (Caporale, 1995). The small molecule metabolites that provide the organisms with adaptive advantages in specific ecological niches are often synonymous to SMs. A typical chemical ecology study often involves the isolation and structural characterization of the putative molecules involved in a given ecological interactions. The molecules are then used to test out the proposed function. The advantage of studying the function of genes and molecules in an ecological context is that they often provide important clues to their natural functions.

The SM biosynthetic genes that encode the production of SMs that mediate ecological interactions are essentially a subset of ecologically important genetic traits. Hence, studies in chemical ecology and ecological genomics have significant overlap and can be highly synergistic. Indeed, molecular genetics and genomics are also transforming the microbial SM (natural product) field, shifting the focus towards understanding biosynthesis and genes-to-molecules relationship (Walsh and Fischbach, 2010) and genomics-guided natural product discovery (Challis, 2008). Bringing these different, but related, fields together will help us bridge the gaps between genes, molecules, and functions.

The cereal pathosystems are perfect test beds for this multipronged chemical ecogenomics approach. Besides the large number of uncharacterized SM gene clusters in the genome, many of them are amenable to genetic manipulation and have established virulence assays (e.g., whole plant and detached leaf assays). An outline of how this emerging approach can be employed to study the SMs in cereal pathogens is presented in Figure 1. We will use some recent ecogenomics/pathogenomics studies in plant pathogens to illustrate the prospect of employing this strategy for understanding the SM functions in these pathogens and for bioactive molecule discovery.

\section{GENOME EVOLUTIONARY DYNAMICS OF SM BIOSYNTHETIC GENES IN FUNGAL PHYTOPATHOGENS}

Secondary metabolite gene clusters are often not essential for the growth and survival of fungi under ideal conditions, but they confer selective advantages on the organisms by producing SMs that may act as defense molecules, signals, siderophores, or

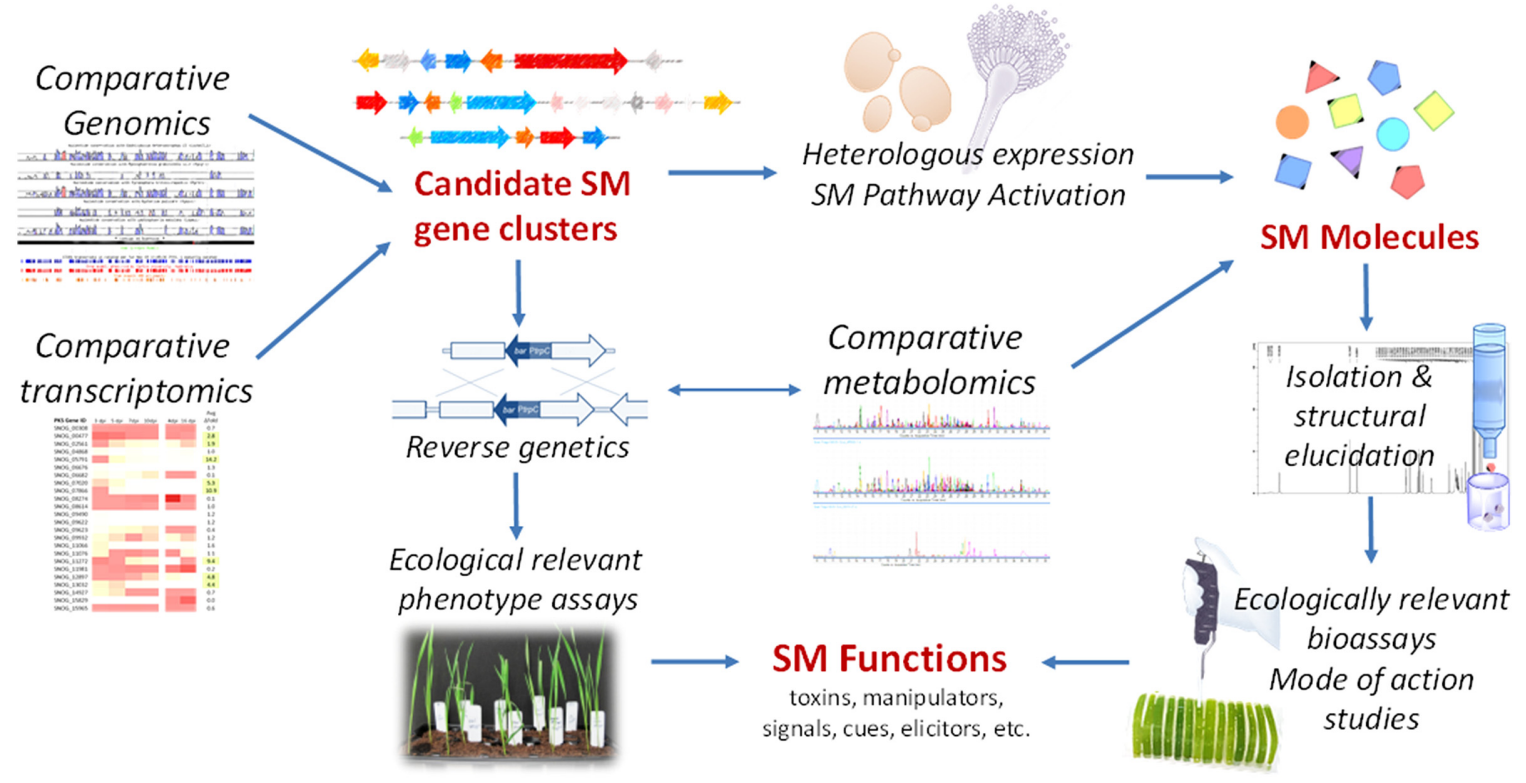

FIGURE 1 | An integrated chemical ecogenomic approach for understanding the functions of secondary metabolites (SMs) and bioactive molecule discovery. The strategy incorporates the common tools in ecological genomics, genome mining and chemical ecology. 
modulators in parasitic/endophytic interactions (Fox and Howlett, 2008; Collemare and Lebrun, 2011; Kusari et al., 2012). Thus, they are often subjected to adaptive evolution via a combination of genetic drifts and natural selection. Evolution mechanisms by gene loss, genetic mutation, gene duplication and divergence, genome rearrangement, fragment recombination and horizontal gene transfer (HGT) are commonly observed among SM gene clusters (Carbone et al., 2007; Patron et al., 2007; Fischbach et al., 2008; Proctor et al., 2009). The evolutionary dynamics of these SM gene clusters combined with the bio-ecological knowledge of the organisms can thus be used to infer the importance of individual SM gene clusters in environmental adaptations. Similar approaches have been recently employed to identify candidate effectors in plant pathogens (Stukenbrock et al., 2010; Gardiner et al., 2012; Ohm et al., 2012; Condon et al., 2013; Manning et al., 2013; Syme et al., 2013).

\section{INTRASPECIFIC COMPARATIVE GENOMICS - SPOT THE DIFFERENCES}

With ready accesses to microbial genome sequencing enabled by next-generation sequencing technologies, genome sampling and re-sequencing is becoming a routine. In specific reference to cereal and other crop pathogens, comparative genomics and phylogenomics analyses on different strains of the same species with different host range or virulence profiles may reveal SM gene clusters that are important to virulence or pathogenicity. Using this approach, Brandon et al. have identified candidate SM gene clusters that may play a role in the virulence of Cochliobolus spp. (Condon et al., 2013). In one of the examples from the study, phylogenomic comparison of non-ribosomal peptide synthetase (NRPS) genes between pathotype 2 (isolate ND90Pr) and pathotype 0 (isolate ND93-1) of the barley pathogen Cochliobolus sativus revealed a NRPS gene cluster that is unique to pathotype 2. Deletion of one of the two NRPS genes in the gene cluster significantly reduced the virulence of Cochliobolus sativus pathotype 2 on barley cultivar Bowman. However, the SM molecule(s) encoded by this gene cluster remains to be identified. As a proof of concept, the authors further demonstrated the utility of this comparative phylogenomic approach to pull out the two polyketide synthase (PKS) genes previously identified to be involved in the host-specific T-toxin biosynthesis in Cochliobolus heterostrophus race T strains (Baker et al., 2006). As expected, the two PKS genes are present in all race $\mathrm{T}$ strains but absent in all race $\mathrm{O}$ strains (Condon et al., 2013). Comparative genomics of the wheat pathogen Parastagonospora nodorum SN15 strain with virulent and avirulent strains have also identified a significant number of strain-specific genes and genomic regions (Syme et al., 2013). Among those is a SM gene cluster that is absent in the genome of the avirulent SN79 strain but present in the two other virulent strains. Based on microarray data from a previous study (Ipcho et al., 2012), the gene cluster appeared to be up-regulated exclusively in planta during wheat leaf infection (Figure 2). Although it remains to be confirmed, it is tempting to speculate the gene cluster may encode a SM that plays a role in virulence.

\section{INTERSPECIFIC COMPARATIVE GENOMICS - SPOT THE SIMILARITIES}

Horizontal gene transfer has now been recognized to be a common phenomenon among fungi (Fitzpatrick, 2012). In crop

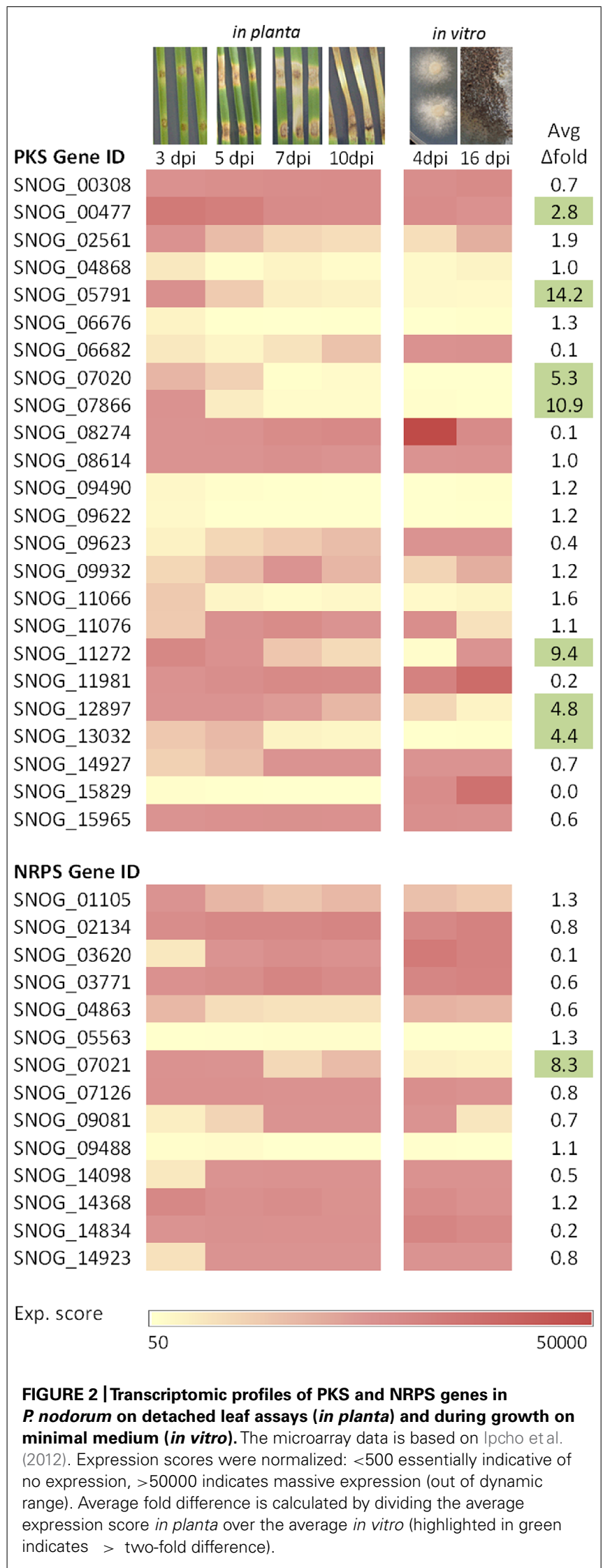


pathogens, HGT is thought to be a major evolutionary force that drives the emergence of new fungal crop diseases (Oliver and Solomon, 2008; Mehrabi et al., 2011). A well-known example is the interspecific horizontal transfer of the ToxA effector gene from $P$. nodorum to Pyrenophora tritici-repentis, resulting in the emergence of the tan-spot disease on wheat (Friesen et al., 2006). HGT of whole or partial SM gene clusters have also been proposed (Patron et al., 2007; Slot and Rokas, 2011; Wight et al., 2013). In a more extreme example, horizontal transfer of whole supernumerary chromosomes containing HST biosynthetic gene clusters has been proposed to confer pathogenicity to different pathotypes of Alternaria alternata (Akagi et al., 2009; Mehrabi et al., 2011). In fact, horizontal transfer has been proposed to be a principal driving force behind the evolution of clustering of SM biosynthetic genes (Walton, 2000). On the other hand, in some cases, the absence/presence of some SM gene clusters between closely related species can be explained by the loss of the gene clusters via genetic drifts (Chooi et al., 2010).

Given that the survival of a SM gene cluster is relying on its ability to confer advantages to the organism, we can expect that the conservation of a SM cluster across multiple species that share similar ecological niches may play similar bio-ecological roles. A good example is the recent discovery of immunosuppressive compounds from human pathogenic fungi (Chooi et al., 2013). Homology searches and comparative genomics identified a homologous gene cluster that is present among Aspergillus fumigatus, Neosartorya fischeri, and six dermatophytic fungi. The conserved gene clusters were demonstrated to produce the immunosuppressive compound neosartoricin (Chooi et al., 2013; Yin et al., 2013).

An example from the cereal pathogens is the SM gene cluster from Cochliobolus carbonum that encodes production of the hostselective HC-toxin required for pathogenicity to $\mathrm{hmhm}$ maize. Interspecific comparative genomic analysis identified HC-toxin gene cluster is present in another maize pathogen Setosphaeria turcica (Condon et al., 2013). Interestingly, the HC-toxin gene cluster is also present in another plant-associated fungus, Alternaria jesenskae, which does not appear to be pathogenic to most plants (Wight et al., 2013). Comparative phylogenomic analysis of NRPS genes among plant pathogens has also revealed that some NRPS genes have undergone recombination and modular rearrangement (Bushley and Turgeon, 2010). For example, modules 1 of ChNPS1 and ChNPS3 from Cochliobolus heterostrophus share high similarity to the modules in the AM-toxin synthetase of Alternaria alternata (Johnson et al., 2000), but other modules of ChNPS1 and ChNPS3 group with other cyclic peptide NRPSs and mono/bi-modular NRPSs in the phylogenetic analysis (Bushley and Turgeon, 2010). In such cases, care has to be taken in interpreting NRPS homology across different species as such NRPS module rearrangements will resulted in different SM products.

In $P$. nodorum, our detailed analysis of the SM gene clusters revealed several genes encoding PKSs and NRPSs that are highly conserved ( $\geq 70 \%$ protein identity) among several dothideomycete cereal pathogens, such as Cochliobolus spp., Leptosphaeria maculans, and Pyrenophora tritici-repentis (Chooi et al., 2014b). One common PKS gene among $P$. nodorum, $P$. tritici-repentis, and
L. maculans has been shown to be responsible for the production of an antifungal compound phomenoic acid in L. maculans (Elliott et al., 2013). Phomenoic acid was proposed to be an antifungal substance used by the pathogens to outcompete other fungi in their environment.

\section{TRANSCRIPTOME DYNAMICS OF SM BIOSYNTHETIC GENES IN ECOLOGICAL INTERACTIONS}

The production of SMs in fungi is highly regulated and often in response to specific biotic interactions and environmental perturbations (Keller et al., 2005; Brakhage, 2013). Therefore, the temporal and spatial expression of SM gene clusters may provide clues to the natural function of the encoded SM molecules in fungi. Traditionally, reverse transcriptase-PCR (RT-PCR) is used to monitor the expression of multiple backbone biosynthetic genes (e.g., PKS and NRPS genes) in fungi, while microarray allows the profiling of global transcriptome dynamics. Recent availability of next-generation RNA-Seq technologies has revolutionized transcriptomic profiling. Unlike microarray, RNA-Seq is not dependent on gene annotations and can provide information about transcript splicing as well. RNA-Seq also allows the simultaneous quantification of transcripts from more than one organism and is thus perfectly suited for the study of organismal interactions. For plant pathogens, these transcriptomic tools can be used to probe the expression of SM gene clusters during various stages of infection. Similar approaches can also be employed to probe fungal-fungal and fungal-bacterial interactions.

A classic example of SM gene cluster that is specifically expressed during host-fungus interactions is the ACE1 PKS-NRPS hybrid gene cluster from the rice pathogen Magnaporthe grisea (Collemare et al., 2008). The expression of ACE1 gene in M. grisea is highly up-regulated during the penetration into the host plant and the protein was localized to appressoria specifically. The gene ace1 confers avirulence toward rice cultivar Pi33 carrying a corresponding resistance $(R)$ gene (Bohnert et al., 2004). ACE1 is likely to play a role in infection or manipulation of the host cell. Nonetheless, the identity of the SM product of ACE1 gene cluster and its function in M. grisea remain to be identified.

Recent global transcriptomic studies of plant pathogens have revealed several SM gene clusters that were expressed during infection. A remarkable example that highlighted the possible roles of fungal pathogen SMs in planta can be found in a recent RNASeq-based transcriptome study of Colletotrichum higginsianum (O'Connell et al., 2012). As many as 12 SM gene clusters (out of 39) were up-regulated before appressorial penetration and during biotrophic phase, but down-regulated during the necrotrophic stage. Similar observations were made on Colletotrichum orbiculare (Gan et al., 2013) and Magnaporthe oryzae (Soanes et al., 2012). Since the plant host remains healthy and asymptomatic during the biotrophic phase, the authors reasoned that the encoded SMs are unlikely to function as phytotoxins but perhaps as small molecule effectors that manipulate the host cells in ways that benefit the fungus or facilitate infection. Fungal SMs are often being screened for phytotoxic activities but their roles in biotrophy of fungi are largely unexplored and warrant further investigation. 
To gain some insights in to the expression pattern of SM gene clusters in necrotrophic pathogens, here, we extracted the previous microarray data of $P$. nodorum during wheat leaf infection (Ipcho et al., 2012). During in planta stage, eight PKS genes and one NRPS genes, out of 24 and 14, respectively, were on average up-regulated twofold or more (Figure 2). There are also several genes that are up-regulated at the end of the necrotrophic phase ( 7 day post inoculation, dpi) before switching to saprotrophy. Close homologs of some of these genes can be found in other plant pathogens. For example, SNOG_05791, which was highly up-regulated at 3 dpi in planta, exhibits $82 \%$ head-to-tail protein identity to the alternapyrone synthase PKSN in A. alternata (Fujii et al., 2005). The final SM product and function of the PKSN gene cluster is yet to be characterized in A. alternata and it would be interesting to determine if the encoded metabolites play a role in the virulence of $P$. nodorum and A. alternata. We are in the process of teasing out the SMs encoded by these candidate gene clusters.

\section{BRIDGING THE GAPS BETWEEN GENES, MOLECULES AND FUNCTIONS}

Ecological genomics is a powerful approach for inferring functionality and narrowing down ecologically relevant SM gene clusters. However, to obtain deeper insights into the bio-ecological functions of these SM gene clusters, the encoded SM molecules have to be first identified. Traditional chemical ecology studies often involved fractionation of an active crude extract guided by ecologically relevant bioassays (e.g., growth inhibition or behavioral response of an interacting organism). However, this method is not suitable if the compounds are only produced in response to specific biotic interactions. Modern metabolomic techniques have enabled the measurement of the metabolome dynamics of organisms during interactions (Prince and Pohnert, 2010). Nonetheless, in systems involved intimate physical interactions between two organisms (e.g., plant-fungal interactions), it is often difficult to track the origin of the metabolites. Furthermore, the amount of compounds that can be obtained through such interaction studies is often limited, which hinders further molecular characterization and mode of action studies.

Genome mining is increasingly becoming a popular approach for natural product discovery in recent years (Challis, 2008; Wiemann and Keller, 2014). To obtain the SMs from a candidate gene cluster, the SM pathway can either be expressed in various heterologous hosts after reconstructing the pathway with host-compatible regulatory elements or in the native producer via consecutive promoter replacements (Ahuja et al., 2012; Lim etal., 2012; Tsunematsu etal., 2013; Lazarus et al., 2014; Yaegashi et al., 2014). Alternatively, the expression of a silent SM gene cluster can be activated by overexpression of pathwayspecific transcriptional regulator, if such regulator is present in the candidate SM gene cluster (Bergmann et al., 2007; Brakhage and Schroeckh, 2011). These methods are capable of producing the desired SM compounds in significant quantities and at the same time establish the important link between genes and molecules. The ready access to genome sequencing means that the availability of DNA sequences is no longer a bottleneck. However, translating SM gene clusters to small molecule products, via the above mentioned methods, remains a time-consuming endeavor. So far, genome mining efforts are focusing on (1) identifying the SM gene cluster of a known compound, (2) discovering analogous SMs from gene clusters that share homology to characterized ones, and (3) untargeted genome mining motivated by the quest to understand gene-to-molecule relationships. The application of ecological genomic tools adds a new dimension to genome mining and will help us navigate the vast genomic information in search for bioactive molecules (Figure 1).

Traditional reverse genetics and mutant phenotyping (i.e., virulence of mutants against the plant host) will still play important roles in casting light on the potential function of the SMs (Figure 1). For example, the presence and absence of specific compound in the wild type and mutant during inter-organism interactions can be verified by metabolic profiling. On the other hand, the information obtained from comparative phenotype assays between mutant and wild type will facilitate the development of specific bioassays to test the hypothetical function of the compounds obtained by ecogenomics-guided SM gene cluster mining. More recently, using this multi-pronged strategy, we have identified the SNOG_00477 PKS gene that is strongly expressed in planta to encode $(R)$-mellein production in $P$. nodorum by gene disruption and heterologous expression in yeast (Chooi etal., 2014a). We further demonstrated that mellein is a strong inhibitor of wheat seed germination. In conclusion, we believe this integrated chemical ecogenomic approach will accelerate the bridging of gaps between genes, molecules and functions, effectively linking genotype-to-phenotype. This multi-pronged approach is also applicable to other microbial ecological systems and will propel the next frontiers in fungal SM research.

\section{ACKNOWLEDGMENTS}

Yit-Heng Chooi is supported by an Australian Research Council (ARC) Discovery Early Career Researcher Award (DECRA) fellowship. Peter S. Solomon is an ARC Future Fellow.

\section{REFERENCES}

Ahuja, M., Chiang, Y. M., Chang, S. L., Praseuth, M. B., Entwistle, R., Sanchez, J. F., et al. (2012). Illuminating the diversity of aromatic polyketide synthases in Aspergillus nidulans. J. Am. Chem. Soc. 134, 8212-8221. doi: 10.1021/ ja3016395

Akagi, Y., Akamatsu, H., Otani, H., and Kodama, M. (2009). Horizontal chromosome transfer, a mechanism for the evolution and differentiation of a plant-pathogenic fungus. Eukaryot. Cell 8, 1732-1738. doi: 10.1128/EC.00135-09 $\mathrm{Au}, \mathrm{T}$. K., Chick, W. S., and Leung, P. C. (2000). The biology of ophiobolins. Life Sci. 67, 733-742. doi: 10.1016/S0024-3205(00)00668-8

Baker, S. E., Kroken, S., Inderbitzin, P., Asvarak, T., Li, B. Y., Shi, L., et al. (2006). Two polyketide synthase-encoding genes are required for biosynthesis of the polyketide virulence factor, T-toxin, by Cochliobolus heterostrophus. Mol. Plant Microbe Interact. 19, 139-149. doi: 10.1094/MPMI-19-0139

Bergmann, S., Schumann, J., Scherlach, K., Lange, C., Brakhage, A. A., and Hertweck, C. (2007). Genomics-driven discovery of PKS-NRPS hybrid metabolites from Aspergillus nidulans. Nat. Chem. Biol. 3, 213-217. doi: 10.1038/nchembio869

Bergstrom, J. D., Dufresne, C., Bills, G. F., Nallin-Omstead, M., and Byrne, K. (1995). Discovery, biosynthesis, and mechanism of action of the zaragozic acids: potent inhibitors of squalene synthase. Annu. Rev. Microbiol. 49, 607-639. doi: 10.1146/annurev.mi.49.100195.003135

Bills, G. F., Gloer, J. B., and An, Z. (2013). Coprophilous fungi: antibiotic discovery and functions in an underexplored arena of microbial defensive mutualism. Curr. Opin. Microbiol. 16, 549-565. doi: 10.1016/j.mib.2013.08.001 
Bohnert, H. U., Fudal, I., Dioh, W., Tharreau, D., Notteghem, J. L., and Lebrun, M. H. (2004). A putative polyketide synthase/peptide synthetase from Magnaporthe grisea signals pathogen attack to resistant rice. Plant Cell 16, 2499-2513. doi: $10.1105 /$ tpc. 104.022715

Brakhage, A. A. (2013). Regulation of fungal secondary metabolism. Nat. Rev. Microbiol. 11, 21-32. doi: 10.1038/nrmicro2916

Brakhage, A. A., and Schroeckh, V. (2011). Fungal secondary metabolites strategies to activate silent gene clusters. Fungal Genet. Biol. 48, 15-22. doi 10.1016/j.fgb.2010.04.004

Bushley, K. E., and Turgeon, B. G. (2010). Phylogenomics reveals subfamilies of fungal nonribosomal peptide synthetases and their evolutionary relationships. BMC Evol. Biol. 10:26. doi: 10.1186/1471-2148-10-2614712148-10-26

Caporale, L. H. (1995). Chemical ecology: a view from the pharmaceutical industry. Proc. Natl. Acad. Sci. U.S.A. 92, 75-82. doi: 10.1073/pnas.92.1.75

Carbone, I., Ramirez-Prado, J. H., Jakobek, J. L., and Horn, B. W. (2007). Gene duplication, modularity and adaptation in the evolution of the aflatoxin gene cluster. BMC Evol. Biol. 7:111. doi: 10.1186/1471-2148-7-111

Challis, G. L. (2008). Mining microbial genomes for new natural products and biosynthetic pathways. Microbiology 154, 1555-1569. doi: 10.1099/mic.0.2008/018523-0

Chooi, Y. H., Cacho, R., and Tang, Y. (2010). Identification of the viridicatumtoxin and griseofulvin gene clusters from Penicillium aethiopicum. Chem. Biol. 17, 483-494. doi: 10.1016/j.chembiol.2010.03.015

Chooi, Y. H., Fang, J., Liu, H., Filler, S. G., Wang, P., and Tang, Y. (2013). Genome mining of a prenylated and immunosuppressive polyketide from pathogenic fungi. Org. Lett. 15, 780-783. doi: 10.1021/ol303435y

Chooi, Y. H., Krill, C., Barrow, R. A., Chen, S., Trengove, R., Oliver, R. P., et al (2014a). An in planta-expressed polyketide synthase produces (R)-mellein in the wheat pathogen Parastagonospora nodorum. Appl. Environ. Microbiol. doi: 10.1128/AEM.02745-14 [Epub ahead of print].

Chooi, Y. H., Muria-Gonzalez, M. J., and Solomon, P. S. (2014b). A genome-wide survey of the secondary metabolite biosynthesis genes in the wheat pathogen Parastagonospora nodorum. Mycology 5, 192-206. doi: 10.1080/21501203.2014.928386

Collemare, J., and Lebrun, M.-H. (2011). "Fungal secondary metabolites: ancient toxins and novel effectors in plant-microbe interactions," in Effectors in Plant Microbe Interactions, eds F. Martin and S. Kamoun (Oxford: Wiley-Blackwell), 379-402.

Collemare, J., Pianfetti, M., Houlle, A. E., Morin, D., Camborde, L., Gagey, M. J., et al. (2008). Magnaporthe grisea avirulence gene ACE1 belongs to an infectionspecific gene cluster involved in secondary metabolism. New Phytol. 179, 196-208. doi: 10.1111/j.1469-8137.2008.02459.x

Condon, B. J., Leng, Y., Wu, D., Bushley, K. E., Ohm, R. A., Otillar, R., et al. (2013). Comparative genome structure, secondary metabolite, and effector coding capacity across Cochliobolus pathogens. PLoS Genet 9:e1003233. doi: 10.1371/journal.pgen.1003233

Elliott, C. E., Callahan, D. L., Schwenk, D., Nett, M., Hoffmeister, D., and Howlett B. J. (2013). A gene cluster responsible for biosynthesis of phomenoic acid in the plant pathogenic fungus, Leptosphaeria maculans. Fungal Genet. Biol. 53, 50-58. doi: 10.1016/j.fgb.2013.01.008

Fischbach, M. A., Walsh, C. T., and Clardy, J. (2008). The evolution of gene collectives: how natural selection drives chemical innovation. Proc. Natl. Acad. Sci. U.S.A. 105, 4601-4608. doi: 10.1073/pnas.0709132105

Fitzpatrick, D. A. (2012). Horizontal gene transfer in fungi. FEMS Microbiol. Lett. 329, 1-8. doi: 10.1111/j.1574-6968.2011.02465.x

Fox, E. M., and Howlett, B. J. (2008). Secondary metabolism: regulation and role in fungal biology. Curr. Opin. Microbiol. 11, 481-487. doi: 10.1016/j.mib.2008.10.007

Friesen, T. L., Stukenbrock, E. H., Liu, Z., Meinhardt, S., Ling, H., Faris, J. D., etal. (2006). Emergence of a new disease as a result of interspecific virulence gene transfer. Nat. Genet. 38, 953-956. doi: 10.1038/ ng1839

Fujii, I., Yoshida, N., Shimomaki, S., Oikawa, H., and Ebizuka, Y. (2005). An iterative type I polyketide synthase PKSN catalyzes synthesis of the decaketide alternapyrone with regio-specific octa-methylation. Chem. Biol. 12, 1301-1309. doi: 10.1016/j.chembiol.2005.09.015

Gan, P., Ikeda, K., Irieda, H., Narusaka, M., O'connell, R. J., Narusaka, Y., etal. (2013). Comparative genomic and transcriptomic analyses reveal the hemibiotrophic stage shift of Colletotrichum fungi. New Phytol. 197, 1236-1249. doi: 10.1111/nph.12085

Gardiner, D. M., Mcdonald, M. C., Covarelli, L., Solomon, P. S., Rusu, A. G., Marshall, M., et al. (2012). Comparative pathogenomics reveals horizontally acquired novel virulence genes in fungi infecting cereal hosts. PLoS Pathog. 8:e1002952. doi: 10.1371/journal.ppat.1002952

Haenni, A. L., Robert, M., Vetter, W., Roux, L., Barbier, M., and Lederer, E. (1965). Structure chimique des aspergillomarasmines A et B. Helv. Chim. Acta 48, 729750. doi: 10.1002/hlca.19650480409

Ipcho, S. V., Hane, J. K., Antoni, E. A., Ahren, D., Henrissat, B., Friesen, T. L., et al. (2012). Transcriptome analysis of Stagonospora nodorum: gene models, effectors, metabolism and pantothenate dispensability. Mol. Plant Pathol. 13, 531-545. doi: 10.1111/j.1364-3703.2011.00770.x

Johnson, R. D., Johnson, L., Itoh, Y., Kodama, M., Otani, H., and Kohmoto, K. (2000). Cloning and characterization of a cyclic peptide synthetase gene from Alternaria alternata apple pathotype whose product is involved in AMtoxin synthesis and pathogenicity. Mol. Plant Microbe Interact. 13, 742-753. doi: 10.1094/MPMI.2000.13.7.742

Keller, N. P., Turner, G., and Bennett, J. W. (2005). Fungal secondary metabolism from biochemistry to genomics. Nat. Rev. Microbiol. 3, 937-947. doi: 10.1038/nrmicro1286

Kempken, F., and Rohlfs, M. (2010). Fungal secondary metabolite biosynthesis - a chemical defence strategy against antagonistic animals? Fungal Ecol. 3, 107-114. doi: 10.1016/j.funeco.2009.08.001

King, A. M., Reid-Yu, S. A., Wang, W., King, D. T., De Pascale, G., Strynadka, N. C., et al. (2014). Aspergillomarasmine A overcomes metallo-beta-lactamase antibiotic resistance. Nature 510, 503-506. doi: 10.1038/nature13445nature13445

Kusari, S., Hertweck, C., and Spiteller, M. (2012). Chemical ecology of endophytic fungi: origins of secondary metabolites. Chem. Biol. 19, 792-798. doi: 10.1016/j.chembiol.2012.06.004

Lazarus, C. M., Williams, K., and Bailey, A. M. (2014). Reconstructing fungal natural product biosynthetic pathways. Nat. Prod. Rep. 31, 1339-1347. doi: 10.1039/ c4np00084f

Lim, F. Y., Sanchez, J. F., Wang, C. C., and Keller, N. P. (2012). Toward awakening cryptic secondary metabolite gene clusters in filamentous fungi. Methods Enzymol. 517, 303-324. doi: 10.1016/B978-0-12-404634-4.00015-12

Manning, V. A., Pandelova, I., Dhillon, B., Wilhelm, L. J., Goodwin, S. B., Berlin, A. M., et al. (2013). Comparative genomics of a plant-pathogenic fungus, Pyrenophora tritici-repentis, reveals transduplication and the impact of repeat elements on pathogenicity and population divergence. G3 (Bethesda) 3, 41-63. doi: 10.1534/g3.112.004044

Mehrabi, R., Bahkali, A. H., Abd-Elsalam, K. A., Moslem, M., Ben M'barek, S., Gohari, A. M., et al. (2011). Horizontal gene and chromosome transfer in plant pathogenic fungi affecting host range. FEMS Microbiol. Rev. 35, 542-554. doi: 10.1111/j.1574-6976.2010.00263.x

Mobius, N., and Hertweck, C. (2009). Fungal phytotoxins as mediators of virulence. Curr. Opin. Plant Biol. 12, 390-398. doi: 10.1016/j.pbi.2009. 06.004

Muria-Gonzalez, M. J., Chooi, Y. H., Breen, S., and Solomon, P. S. (2014). The past, present and future of secondary metabolite research in the Dothideomycetes. Mol. Plant Pathol. doi: 10.1111/mpp.12162 [Epub ahead of print].

O’Connell, R. J., Thon, M. R., Hacquard, S., Amyotte, S. G., Kleemann, J., Torres, M. F., et al. (2012). Lifestyle transitions in plant pathogenic Colletotrichum fungi deciphered by genome and transcriptome analyses. Nat. Genet. 44, 1060-1065. doi: 10.1038/ng.2372

Ohm, R. A., Feau, N., Henrissat, B., Schoch, C. L., Horwitz, B. A., Barry, K. W., et al. (2012). Diverse lifestyles and strategies of plant pathogenesis encoded in the genomes of eighteen Dothideomycetes fungi. PLoS Pathog. 8:e1003037. doi: 10.1371/journal.ppat.1003037

Oliver, R. P., and Solomon, P. S. (2008). Recent fungal diseases of crop plants: is lateral gene transfer a common theme? Mol. Plant Microbe Interact. 21, 287-293. doi: 10.1094/MPMI-21-3-0287

Patron, N. J., Waller, R. F., Cozijnsen, A. J., Straney, D. C., Gardiner, D. M., Nierman, W. C., et al. (2007). Origin and distribution of epipolythiodioxopiperazine (ETP) gene clusters in filamentous ascomycetes. BMC Evol. Biol. 7:174. doi: 10.1186/1471-2148-7-174

Pompe, S., Simon, J., Wiedemann, P. M., and Tannert, C. (2005). Future trends and challenges in pathogenomics. A Foresight study. EMBO Rep. 6, 600-605. doi: 10.1038/sj.embor.7400472 
Prince, E. K., and Pohnert, G. (2010). Searching for signals in the noise: metabolomics in chemical ecology. Anal. Bioanal. Chem. 396, 193-197. doi: 10.1007/s00216-009-3162-5

Proctor, R. H., Mccormick, S. P., Alexander, N. J., and Desjardins, A. E. (2009). Evidence that a secondary metabolic biosynthetic gene cluster has grown by gene relocation during evolution of the filamentous fungus Fusarium. Mol. Microbiol. 74, 1128-1142. doi: 10.1111/j.1365-2958.2009.06927.x

Rohlfs, M., and Churchill, A. C. L. (2011). Fungal secondary metabolites as modulators of interactions with insects and other arthropods. Fungal Genet. Biol. 48, 23-34. doi: 10.1016/j.fgb.2010.08.008

Scherlach, K., Boettger, D., Remme, N., and Hertweck, C. (2010). The chemistry and biology of cytochalasans. Nat. Prod. Rep. 27, 869-886. doi: 10.1039/b903913a

Schmidt, S. M., and Panstruga, R. (2011). Pathogenomics of fungal plant parasites: what have we learnt about pathogenesis? Curr. Opin. Plant Biol. 14, 392-399. doi: 10.1016/j.pbi.2011.03.006

Slot, J. C., and Rokas, A. (2011). Horizontal transfer of a large and highly toxic secondary metabolic gene cluster between fungi. Curr. Biol. 21, 134-139. doi: 10.1016/j.cub.2010.12.020

Soanes, D. M., Chakrabarti, A., Paszkiewicz, K. H., Dawe, A. L., and Talbot, N. J. (2012). Genome-wide transcriptional profiling of appressorium development by the rice blast fungus Magnaporthe oryzae. PLoS Pathog. 8:e1002514. doi: 10.1371/journal.ppat.1002514

Stergiopoulos, I., Collemare, J., Mehrabi, R., and De Wit, P. J. (2013). Phytotoxic secondary metabolites and peptides produced by plant pathogenic Dothideomycete fungi. FEMS Microbiol. Rev. 37, 67-93. doi: 10.1111/j.1574-6976.2012.00349.x

Stukenbrock, E. H., Jorgensen, F. G., Zala, M., Hansen, T. T., Mcdonald, B. A., and Schierup, M. H. (2010). Whole-genome and chromosome evolution associated with host adaptation and speciation of the wheat pathogen Mycosphaerella graminicola. PLoS Genet 6:e1001189. doi: 10.1371/journal.pgen.1001189

Syme, R. A., Hane, J. K., Friesen, T. L., and Oliver, R. P. (2013). Resequencing and comparative genomics of Stagonospora nodorum: sectional gene absence and effector discovery. G3 (Bethesda) 3, 959-969. doi: 10.1534/g3.112.004994

Tittiger, C. (2004). Functional genomics and insect chemical ecology. J. Chem. Ecol. 30, 2335-2358. doi: 10.1007/s10886-004-7940-4

Tsunematsu, Y., Ishiuchi, K., Hotta, K., and Watanabe, K. (2013). Yeast-based genome mining, production and mechanistic studies of the biosynthesis of fungal polyketide and peptide natural products. Nat. Prod. Rep. 30, 1139-1149. doi: $10.1039 / \mathrm{c} 3 \mathrm{np} 70037 \mathrm{~b}$

Ungerer, M. C., Johnson, L. C., and Herman, M. A. (2008). Ecological genomics: understanding gene and genome function in the natural environment. Heredity 100, 178-183. doi: 10.1038/sj.hdy.6800992
Vizcaino, M. I., Guo, X., and Crawford, J. M. (2014). Merging chemical ecology with bacterial genome mining for secondary metabolite discovery. J. Ind. Microbiol. Biotechnol. 41, 285-299. doi: 10.1007/s10295-013-1356-5

Walsh, C. T., and Fischbach, M. A. (2010). Natural products version 2.0: connecting genes to molecules. J. Am. Chem. Soc. 132, 2469-2493. doi: 10.1021/ja909118a

Walton, J. D. (2000). Horizontal gene transfer and the evolution of secondary metabolite gene clusters in fungi: an hypothesis. Fungal Genet. Biol. 30, 167-171. doi: 10.1006/fgbi.2000.1224

Walton, J. D. (2006). HC-toxin. Phytochemistry 67, 1406-1413. doi: 10.1016/j.phytochem.2006.05.033

Wiemann, P., and Keller, N. P. (2014). Strategies for mining fungal natural products. J. Ind. Microbiol. Biotechnol. 41, 301-313. doi: 10.1007/s10295-013-1366-3

Wight, W. D., Labuda, R., and Walton, J. D. (2013). Conservation of the genes for HC-toxin biosynthesis in Alternaria jesenskae. BMC Microbiol. 13:165. doi: 10.1186/1471-2180-13-165

Yaegashi, J., Oakley, B. R., and Wang, C. C. (2014). Recent advances in genome mining of secondary metabolite biosynthetic gene clusters and the development of heterologous expression systems in Aspergillus nidulans. J. Ind. Microbiol. Biotechnol. 41, 433-442. doi: 10.1007/s10295-013-1386-Z

Yin, W. B., Chooi, Y. H., Smith, A. R., Cacho, R. A., Hu, Y., White, T. C., et al. (2013). Discovery of cryptic polyketide metabolites from dermatophytes using heterologous expression in Aspergillus nidulans. ACS Synth. Biol. 2, 629-634. doi: $10.1021 / \mathrm{sb} 400048 \mathrm{~b}$

Conflict of Interest Statement: The authors declare that the research was conducted in the absence of any commercial or financial relationships that could be construed as a potential conflict of interest.

Received: 26 September 2014; paper pending published: 27 October 2014; accepted: 06 November 2014; published online: 19 November 2014.

Citation: Chooi Y-H and Solomon PS (2014) A chemical ecogenomics approach to understand the roles of secondary metabolites in fungal cereal pathogens. Front. Microbiol. 5:640. doi: 10.3389/fmicb.2014.00640

This article was submitted to Microbial Physiology and Metabolism, a section of the journal Frontiers in Microbiology.

Copyright (C) 2014 Chooi and Solomon. This is an open-access article distributed under the terms of the Creative Commons Attribution License (CC BY). The use, distribution or reproduction in other forums is permitted, provided the original author(s) or licensor are credited and that the original publication in this journal is cited, in accordance with accepted academic practice. No use, distribution or reproduction is permitted which does not comply with these terms. 